\title{
Segmentation of White Matter Lesions from Volumetric MR Images
}

\author{
S. A. Hojjatoleslami, F. Kruggel, and D. Y. von Cramon \\ Max-Planck-Institute of Cognitive Neuroscience \\ Stephanstraße 1A, D-04103 Leipzig, Germany
}

\begin{abstract}
Quantitative analysis of the changes to the brain's white matter is an important objective for a better understanding of pathological changes in various forms of degenerative brain diseases. To achieve an accurate quantification, an algorithm is proposed for automatic segmentation of white matter atrophies and lesions from T1-weighted 3D Magnetic Resonance (MR) images of the head. Firstly, white matter, gray matter and cerebrospinal fluid (CSF) compartments are segmented. Then, external and internal cisterns are separated by placing cutting planes relative to the position of the anterior and posterior commissure. Finally, a region growing method is applied to detect lesions inside the white matter. Since lesions may be adjacent to the gray matter, we use the external cisterns as a clue to prevent the algorithm from absorbing low gray level points in the gray matter.

The method is fully applied to detect the white matter lesions and relevant structures from a set of $41 \mathrm{MR}$ images of normal and pathological subjects. Subjective assessment of the results demonstrates a high performance and reliability of this method.
\end{abstract}

\section{Introduction}

A number of brain diseases lead to focal and diffuse pathologic changes detectable by MR tomography. However, the clinical relevance of these findings with respect to cognitive abilities or impairments of a patient is often unclear. Traditionally, pathologic changes in MR tomograms are assessed visually or using semi-automatic techniques. These measures are compared with behavioral data to assess the clinical relevance of MR findings. While visual inspection and expert rating are still the "gold standard" in MR evaluation, it is hoped that image processing can assist and support this process and yield results which are rater-independent and have a higher reliability.

Extraction of such quantitative measures is concerned with computing a number of descriptors representing the properties of anatomical structures in the brain. The most important step in quantitative analysis is an accurate segmentation of tissues in MR images. The segmentation of tissues has received considerable attention in medical image processing community [1,2,3,4]. Although promising results on large sets of normal images is reported, validation of the 


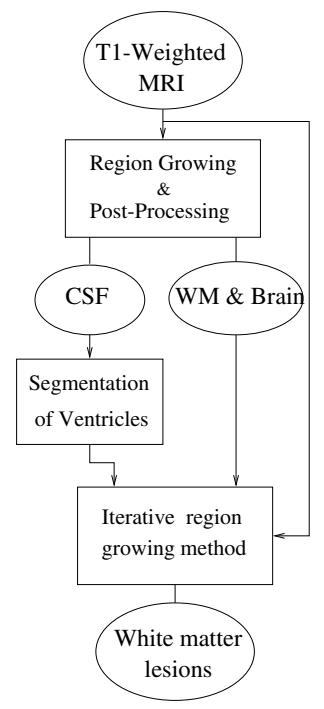

Fig. 1. Different processing steps for segmentation of white matter lesions in MR image.

techniques on pathological datasets remains a persistent problem. Not surprisingly, the problem of white matter lesion detection has also received a considerable interest in the literature where the use of various image analysis techniques including pyramidal approach [5], intensity based [6,7] and model based [8] techniques, have been addressed. The reader is referred to good surveys on general purpose image segmentation techniques $[9,10]$ and those which are specificly designed or optimized for MRI segmentation purpose $[1,2,3,4]$.

WM lesions are one of the main signs of various forms of degenerative brain diseases. In T1-weighted MR images, they appear as faint, dark, arbitrarily shaped blobs, which range from a few millimeters to several centimeters in size. A better understanding of the size, number and the spatial distribution of the lesions may give important clues to the mechanisms of the disease, provide a new tool for monitoring their change over time and allow drawing conclusions about the progression of a disease. A reliable detection of these lesions is difficult: fuzzy lesion boundaries, noise and non-uniformity in MR images produce a severe lack of definition of WM lesions. They may also lead to a reduction of the WM volume, which is also detected indirectly as an increase of the CSF compartment.

In this paper we aim to present a region based algorithm for the segmentation of the white matter, the internal and external CSF compartments and lesions from volumetric T1-weighted MR images of the head. As shown in Figure 1, we first segment the main tissues (WM, GM and CSF) from the MR data using a region growing method. The CSF compartment is split into internal and exter- 
nal cisterns using a heuristic technique. In the final step, the holes within the segmented WM are used as starting points for the region growing method to find the highest gradient boundary for every blob in the white matter. Since there are junctions between the lesions and the cortex, we use the external cisterns as clue to prevent the growing process from covering the low intensity points in the cortex.

This paper is organized as follows. Section2 presents a brief overview of the region growing method followed by a description of various processing steps applied to segment the white matter lesions from the brain. Section 3 considers the capability of the algorithm and evaluates its performance on a large set of normal and pathological datasets. The last section presents summary and conclusion of this study.

\section{Methods}

The method [11] starts from a seed point and absorbs the highest gray level point in its boundary to expand the region. During the growing process, it considers a discontinuity measure, called peripheral contrast, to choose from a set of regions evolved during the growing process, a region with the highest gradient boundary as the final output. The discontinuity measure computes the difference between the gray level average of internal boundary of the region and its current boundary. The "internal boundary" is the boundary produced by the set of connected outermost voxels of the current region and "current boundary" as the set of voxels adjacent to the current region. This algorithm is used in the first processing step to segment the scalp and other signal intense parts of the head.

A modified technique [12] which applies a new criterion is then employed to segment the white matter, gray matter and the CSF. Here, the segmented region in the previous step, called thereafter the mask, is used to prevent the growing process from joining the non-brain parts of the head. The criterion detects the point with the minimum gray level value in the current region which links the brain to the mask. These junctions are detected and blocked to allow the algorithm to consider the lower gray-level points in the local neighborhood without growing into the already segmented mask.

The criterion checks every new candidate point $y_{i}$ with the set of points in the mask $\mathbf{S}$. If the point is inside the mask, $y_{\lambda} \in \mathbf{S}$, the size of the region would reduce to $k$ where $y_{k}=y_{\min }$ is the last minimum gray level point joined to the region. The set of subsequently appended points, with index $i>k+1$, will be labeled as the mask, and the growing process continues by absorbing a new point in the boundary. Such criterion can be formalized using:

$$
y_{i}>y_{k} \quad \text { for } i=k+1, k+2, \ldots, \lambda
$$

where $y_{i}$ is the sequence of pixels joining the region and $y_{\lambda}$ belongs to the mask. 


\subsection{Segmentation of White Matter, Gray Matter and CSF}

To demonstrate the capability of our algorithm for segmentation of white matter, gray matter and CSF, we consider the behavior of the algorithm applied to a 3D MR image shown in Figure 2-top. The mappings of gray level and peripheral contrast for the image for a starting point inside the brain stem is shown in Figure 2-bottom. The gray level mappings are shown for two conditions: (i) using the segmented scalp as a mask and, (2) using no mask and, therefore, ignoring the criterion (1). The mask is segmented using the algorithm when a point inside the scalp is used to start the growing process [11].

Let us consider the sequence of points absorbed by the growing process. If the algorithm is applied without the criterion (1), the region grows to include the white matter and then through bright junctions (i.e. optical nerves) joins the eyes and other signal intense parts of the head. The sharp increase in the gray level at index number $7.7 e+5$ is related to growing into the eyes and the scalp. When the mask is used, the mapping does not show any rise at this point. The criterion blocks the minimum point joining the scalp, and the current region continues to grow inside the brain. Comparing the two gray level mappings, it becomes evident that the gray level values of the new points are decreasing when the mask is used while fluctuations are observed when the mask is not applied. We, therefore, are interested to choose the locally highest gradient boundary by considering the peripheral contrast only when the mask and criterion (1) were used.

The peripheral contrast, see Figure 2-bottom, starts from low values and increases at the beginning of growing process. This rise is related to the relatively high gradient at the boundary of the white matter. The peripheral contrast changes slowly by the gradient inside the gray matter and shows a sharp increase when the gray matter is being covered. It then reduces when the growing process absorbs lower gray level values in the CSF. Because a low gradient inside the CSF is encountered, a clear peak for the brain compartment at index number $1.68 e+6$ is found. The third small peak at index number $2.3 e+6$ is related to the gradient at the boundary of CSF and the dura mater.

We use the three changes in the peripheral contrast as indicators for segmenting white matter, brain and CSF. For our pathological datasets, the white matter typically has a very fuzzy gradient boundary producing only a small change in the peripheral contrast measure. To increase the reliability of WM segmentation, we use the gradient of the peripheral contrast during the growing process and segment the region when a change in the gradient direction is observed. The global peak generated by the high gradient boundary of the brain is used as a reference for separating CSF and WM.

\section{$2.2 \quad$ Post Processing Step}

A post-processing step is applied to remove some thin structures, eg. parts of the optical nerves, veins and dura mater which connect the brain to its outer hulls. Typically, those junctions are very thin and, therefore, can be easily pruned 

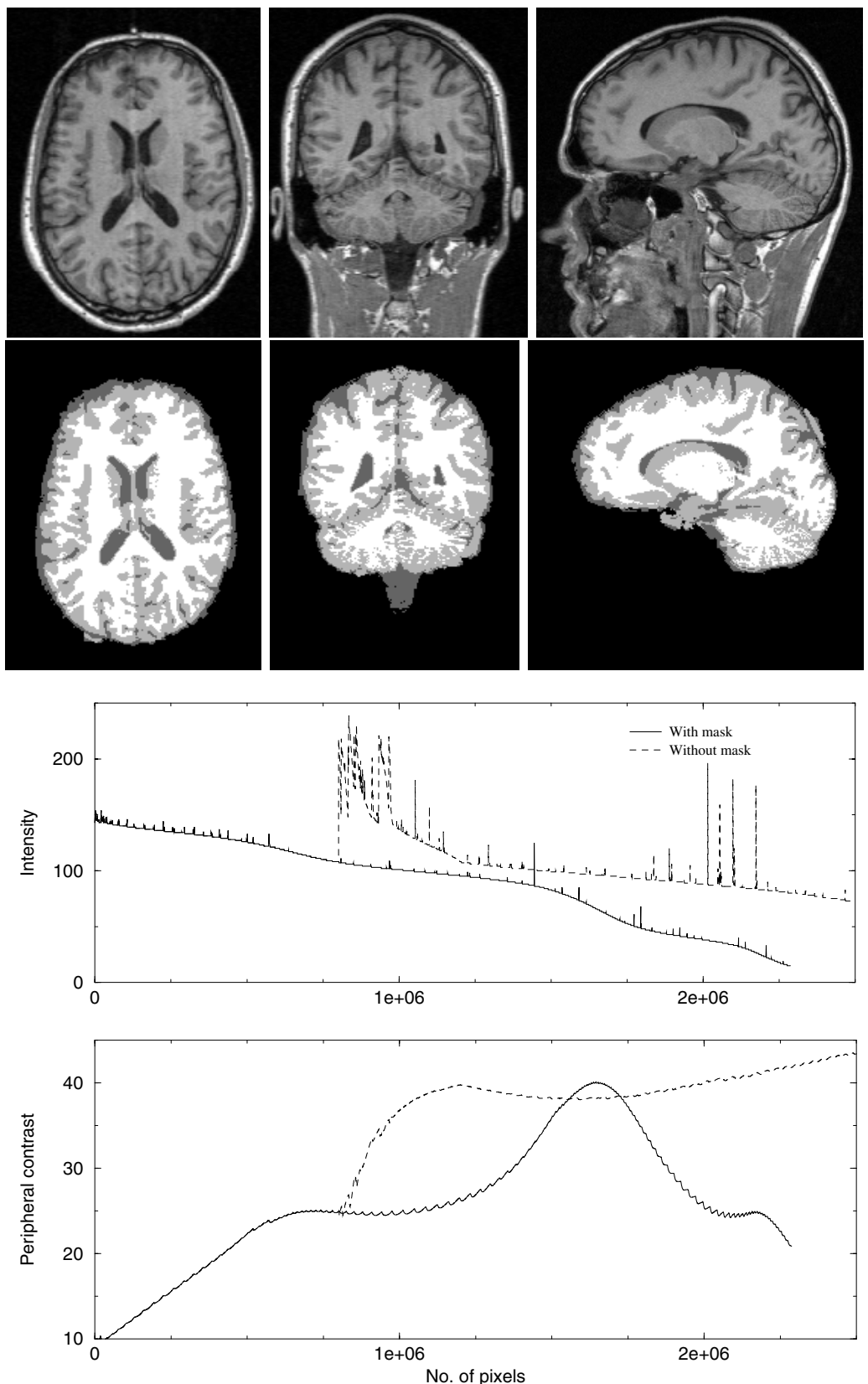

Fig. 2. First row: Orthogonal sections from a 3D brain dataset. Second row: The segmentation of tissues from the MR image (white: white matter, gray: gray matter and dark gray: CSF). The graph shows the gray level and peripheral contrast mappings for the two conditions during the growing process. 
by applying morphological filters [13] with a small structuring element. This is performed using a closing filter followed by a dilation with spherical Structuring elements with diameters of 5 and 3 voxels, respectively.

\subsection{Segmentation of Ventricles}

The inner cisterns mostly contain CSF and are surrounded by white matter. Only small connections exist which link the 3rd ventricle to the external cisterns. Cutting at this junction is expected to separate the external cisterns from the ventricles. The tiny membrane between the $3 \mathrm{rd}$ ventricle and the quadrigeminal cistern is often not fully connected due to the partial volume effect and thus leads to segmentation problems.

Because datasets are aligned with the stereotactical coordinate system, we can simply use the positions of the anterior (CA) and the posterior commissure (CP) to generate suitable cutting plains for separating inner and outer cisterns. The first cut is performed parallel to coronal plane with a thickness of $4 \mathrm{~mm}$, width of $16 \mathrm{~mm}$ (centered at CP) starting from $1 \mathrm{~mm}$ above CA down towards the neck. Two other cuts were performed with a reference from CP: one parallel to the axial plane starting from $8 \mathrm{~mm}$ up and $7 \mathrm{~mm}$ back of the head with the width of $18 \mathrm{~mm}$ towards the neck. A small cut is also performed parallel to the sagittal plane, $3 \mathrm{~mm}$ below $\mathrm{CP}$ with the width of $40 \mathrm{~mm}$ connecting the two previous planes. However, this last cut was not necessary for most of the cases but useful for brains with extreme atrophies.

\subsection{White Matter Lesion Detection}

To detect the small white matter lesions, we use the region growing method starting from the holes inside the segmented white matter. The growing process absorbs the low gray level points in its current boundary thus covering the lesion. The external cisterns are used as a mask to prevent the algorithm from growing into the gray matter or outside the brain. The peripheral contrast is then used to find the highest gradient boundary for each lesion.

An example of the gray level and peripheral contrast for two different lesions is shown in Figure 3. The peripheral contrast starts at a center point and reduces to a minimum value at index numbers 35 and 50. It then gradually approaches the zero level and increases sharply at the final stages of the growing process when most of boundary points are excluded based on criterion (1). This peak is related to very high gray level points (spikes) which will be absorbed at the end of process. The process is terminated when the number of boundary points are very low. The global minimum is used to segment the WM lesions. Limits for a minimum size of 5 voxels and a peripheral contrast of -6 are imposed to exclude false positive detections, which correspond to small and faint regions at resolution limits of the MR scanner. 

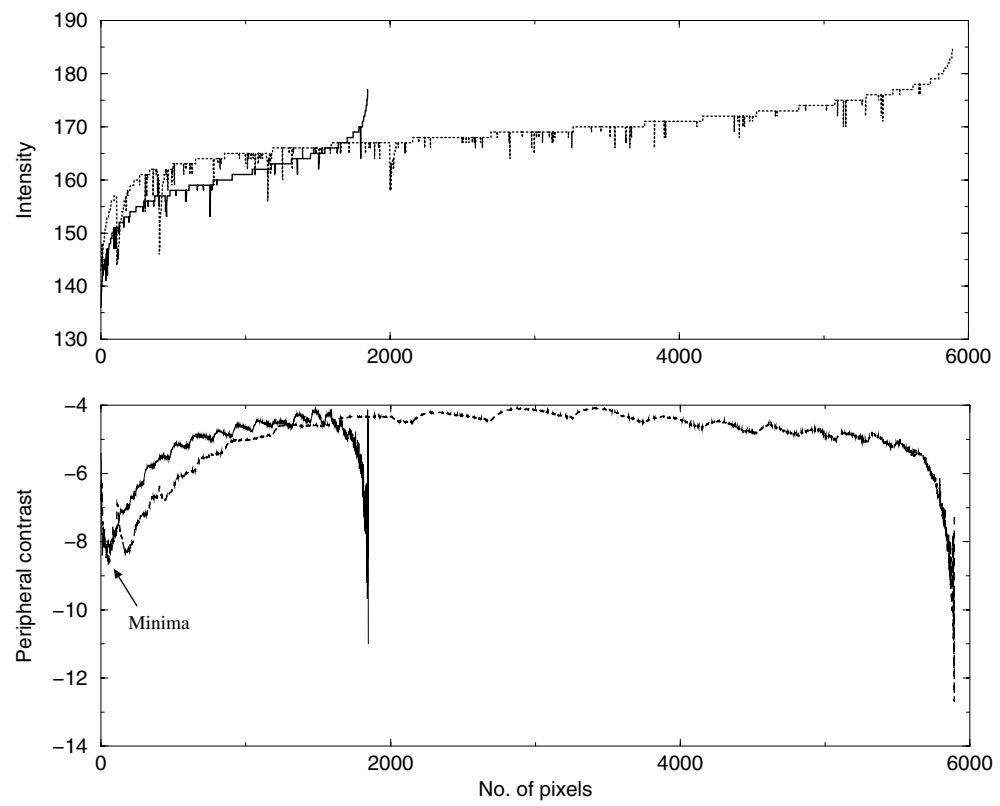

Fig. 3. Gray level and peripheral contrast mappings for two different white matter lesions.

\section{Results}

We used 41 T1-weighted MR images obtained by scanning persons suffering from brain atrophy (possible Alzheimer's disease), white matter disease, and lacunar infarcts. The images were obtained by a 1.5 Tesla clinical MR scanner at the university hospital Leipzig during a routine study of patients. All datasets were aligned with the stereotactical coordinate system before segmentation and their spatial resolution was interpolated to an isotropical resolution of $1 \mathrm{~mm}$.

The two starting points required for the segmentation algorithm were chosen inside the scalp and a bright part of the brain. Segmented tissues after the postprocessing step are shown in Figure 4. WM lesions are shown in white overlayed on the original MR image (top row). Inner cisterns are shown in black, outer cisterns in gray (middle row) with respect to the segmented brain in white.

The results were assessed by a specialist in the field using visual inspection of cross-sections and 3D renderings of the segmented brains. Brain segmentation was very reliable and well in agreement with the specialist. White matter segmentation proved to be more difficult, partly due to the "routine" quality of the data, and partly due to the marked cortical atrophy in some cases. This problem also caused difficulty in detection of the relevant turning point from the peripheral contrast. The CSF and ventricles were segmented quite reliably. In some cases, the heuristic placement of the cutting planes clipped parts of 


\begin{tabular}{|c|c|c|c|c|c|c|c|c|c|c|c|c|c|c|}
\hline \multirow[t]{2}{*}{ Diagnosis } & \multirow{2}{*}{\multicolumn{2}{|c|}{ Age Sex }} & \multirow{2}{*}{\multicolumn{2}{|c|}{$\begin{array}{l}\text { brain } \\
\text { ml \% }\end{array}$}} & \multicolumn{2}{|c|}{$\mathrm{EC}$} & \multicolumn{2}{|c|}{$\mathrm{IC}$} & \multicolumn{2}{|c|}{ WM } & \multicolumn{2}{|c|}{ GM } & \multirow{2}{*}{\multicolumn{2}{|c|}{$\begin{array}{l}\mathrm{LS} \text { LN } \\
\mathrm{ml} \text { no }\end{array}$}} \\
\hline & & & & & $\mathrm{ml}$ & $\%$ & $\mathrm{ml}$ & $\%$ & $\mathrm{ml}$ & $\%$ & $\mathrm{ml}$ & $\%$ & & \\
\hline Mixed Dementia & 81 & $\mathrm{~m}$ & 1331 & 72 & 328 & 17.8 & 180 & 9.8 & 802 & 44 & 529 & 29 & 11.7 & 10 \\
\hline Mixed Dementia & 82 & $\mathrm{f}$ & 1044 & 74 & 341 & 24.1 & 28 & 1.9 & 606 & 43 & 437 & 30 & 10.3 & 45 \\
\hline M. Alzheimer & 84 & $\mathrm{~m}$ & 1245 & 68 & 543 & 29.7 & 43 & 2.4 & 711 & 39 & 533 & 29 & 6.1 & 126 \\
\hline WM Disease & 77 & $\mathrm{f}$ & 1036 & 70 & 395 & 26.8 & 44 & 3.0 & 518 & 35 & 517 & 35 & 8.8 & 63 \\
\hline WM Disease & 75 & $\mathrm{~m}$ & 1104 & 69 & 416 & 26.1 & 72 & 4.6 & 552 & 35 & 552 & 35 & 6.7 & 60 \\
\hline WM Disease & 77 & $\mathrm{f}$ & 1298 & 74 & 437 & 24.9 & 22 & 1.2 & 649 & 37 & 649 & 37 & 9.5 & 90 \\
\hline WM Disease & 77 & $\mathrm{f}$ & 1061 & 70 & 429 & 28.2 & 28 & 1.8 & 532 & 35 & 530 & 35 & 14.1 & 126 \\
\hline Cont & 32 & $\mathrm{~m}$ & 1347 & 82 & 277 & 16.9 & 14 & 0.8 & 660 & 40 & 687 & 42 & 0 & \\
\hline Control & 25 & $\mathrm{~m}$ & 1418 & 81 & 309 & 17.7 & 14 & 0.8 & 699 & 40 & 719 & 41 & 0 & \\
\hline Control & 24 & $\mathrm{~m}$ & 1527 & 83 & 294 & 16.0 & 19 & 1.0 & 763 & 41 & 764 & 41 & 0 & \\
\hline
\end{tabular}

Table 1. Measures of intracranial compartments extracted from our segmentation algorithm for 7 pathological and 3 normal subjects. Volumes of the compartments are given in $\mathrm{cm}^{3}$ and in percentage of the intracranial volume (CSF+brain) are tabulated for the brain, internal cisterns (IC), external cisterns (EC), white matter (WM), gray matter (GM), white matter lesion size (LS) and also the number of white matter lesions (LN).

the 3rd ventricle. However, the error induced hereby was less than $3 \%$ of the total ventricle volume in all cases.

The validation of the white matter lesions is very difficult due to similarity and junctions of the lesions with gray matter. The performance as evaluated on the images were very well in agreement with an independently performed visual inspection by a specialist. Table 1 presents the size and number of white matter lesions for 3 normal subjects and 7 patients.

In comparison to normal subjects, all patients showed a reduction of the GM and the WM compartment (see Table 1), i.e. a global brain atrophy. In the first case, this atrophy led to a substantial increase of the inner cisterns, while in all other cases, only the outer cisterns were enlarged. A rough 1:1 ratio of the GM: WM compartment, which was found for the controls, was maintained for cases 4-7, indicating a global atrophy. For cases 1-3, a substantial atrophy of the GM compartment was detected.

We summarized our findings in Table 2 for patients suffering from Alzheimer's disease, a global brain atrophy in conjunction with a focus on the GM compartment was found, and the external cisterns were enlarged. Patients suffering from a WM disease retained the GM:WM ratio found in controls, although the brain volume was reduced. Both findings may overlap. It is still under study why in some cases only the inner cisterns are enlarged.

The main strength of the algorithm is that it needs only two starting points to segment the structures. The starting points were chosen automatically relative to CA and CP. Acceptable results were achieved on 36 out of 41 images without manual intervention. The starting points for other images were chosen manually. 


\begin{tabular}{lcccc} 
Diagnosis & $\begin{array}{c}\text { Brain } \\
\text { Volume }\end{array}$ & $\begin{array}{c}\text { EC } \\
\text { Comp. }\end{array}$ & $\begin{array}{c}\text { IC } \\
\text { Comp. }\end{array}$ & $\begin{array}{c}\text { GM:WM } \\
\text { Ratio }\end{array}$ \\
\hline M. Alzheimer & $\downarrow$ & $\uparrow$ & $=$ & $\downarrow$ \\
Mixed Dementia & $\downarrow$ & $\uparrow$ & $=, \uparrow$ & $\downarrow$ \\
WM Disease & $\downarrow$ & $\uparrow$ & $=$ & $=$ \\
\hline
\end{tabular}

Table 2. Atrophy patterns for the subgroups of the patients.

\section{Conclusion}

We have proposed a new algorithm for segmentation of white matter lesions from T1-weighted volumetric images of the head. The algorithm applies a region growing algorithm to segment different tissues (WM, GM, CSF) based on changes in the discontinuity measure during the growing process. Inner and outer cisterns are separated by introducing cutting planes. Finally, small holes in the white matter are used as starting points to segment WM lesions. The external cisterns were used to prevent the region growing process from including gray matter in the lesions.

The performance of the technique applied for white matter lesion segmentation from 41 datasets obtained by 1.5-Tesla scanner are very encouraging.

\section{Acknowledgments}

The authors are very grateful to Professor M.J. Gertz from Department of Psychiatry, University Clinic Leipzig, for providing the data.

\section{References}

1. M S Atkins and B T Mackiewich. Fully automatic segmentation of the brain in mri. IEEE Trans Med Imag, 17(1):98-107, 1998. 52, 53

2. J C Bezdek, L O Hall, and L P Clarke. Review of mr image segmentation techniques using pattern recognition. Med Phys, 20(4):1033-1048, 1993. 52, 53

3. L P Clarke, R P Velthuizen, M A Camacho, J J Heine, M Vaidyanathan, L O Hall, R W Thatcher, and M L Silbiger. Mri segmentation: Methods and applications. Magn Reson Imag, 13(3):343-368, 1995. 52, 53

4. J C Rajapakse and F Kruggel. Segmentation of mr images with intensity inhomogeneities. Image and Vision Computing, 16:165-180, 1998. 52, 53

5. C Pachai, Y M Zhu, J Grimaud, M Hermier, A Dromigny-Badin, A Boudraa, G Gimenez, C Confavreux, and J C Froment. A pyramidal approach for automatic segmentation of multiple sclerosis lesions in brain mri. Computerized Med Imag and Graph, 22:399-408, 1998. 53

6. B Johnston and MS Atkins. Segmentation of multiple sclerosis lesions in intensity corrected multispectral mri. IEEE Trans Med Imag, 15(2):154-167, 1996. 53

7. B Udupa, S Wei L Samarasekera, Y Miki, and M A Van Bucchem. Multiple sclerosis lesion quantification using fuzzy-connectness principles. IEEE: Trans on Med Imag, 16(5):598-609, 1997. 53 
8. M Kamber, R Shinghal, L Collins, GS Francis, and A C Evans. Model based 3$\mathrm{d}$ segmentation of multiple sclerosis lesions in magnetic resonance brain images. IEEE Trans Med Imag, 14(3):442-453, 1995. 53

9. R M Haralik and L G Shapiro. Survey: Image segmentation techniques. Comput Vision, Graphics, Image Processing, 29:100-132, 1985. 53

10. N R Pal and S K Pal. A review on image segmentation techniques. Pattern Recognition, 26:1277-1294, 1993. 53

11. S A Hojjatoleslami and J Kittler. Region growing: A new approach. IEEE Trans Image Proc, 7(7):1079-1084, 1998. 54, 55

12. S A Hojjatoleslami, F Kruggel, and D Y von Cramon. A region based algorithm for brain segmentation in mri. submitted, 1999. 54

13. J Serra. Image analysis and mathematical morphology: theoretical advances, volume 2. Academic Press, New York, 1988. 57 

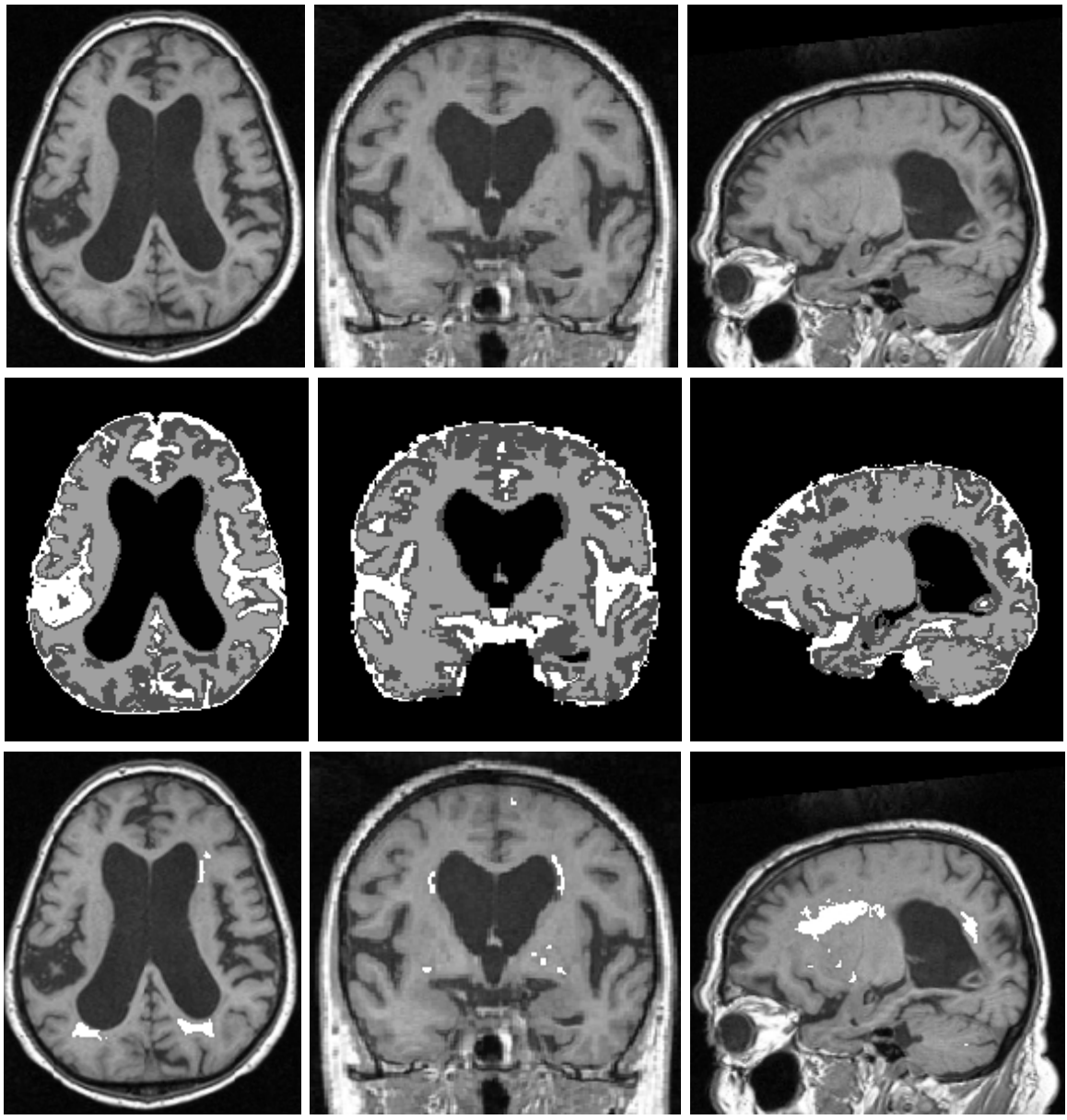

Fig. 4. Different structures segmented by our algorithm from a case of mixed dementia. First row: Original image. Second row: White matter (bright gray), Gray matter (dark gray), internal cisterns (black), external cisterns (white). Third row: White matter lesions (white) overlayed on the original image. 